\title{
Constructive Interference based Joint Combiner and Precoder Design in Multiuser MIMO Systems
}

\author{
Zhongxiang Wei, Member, IEEE, Christos Masouros, Senior Member, IEEE, and Tongyang Xu, Member, IEEE
}

\begin{abstract}
Instead of suppressing multiuser interference as a harmful element by conventional precoders, constructive interference (CI) technique is able to exploit multiuser interference as a beneficial element for enhancing receiver performance. This paper is the first work investigating the feasibility of $\mathrm{CI}$ in multiuser multiple-input and multiple-output (MIMO) systems. A joint CI based combiner and precoder approach is proposed, and then a robust design is further developed taking into consideration of imperfect channel state information (CSI). Simulation shows that the proposed designs significantly outperform the existing benchmarks, and shows a high level of robustness against CSI error.
\end{abstract}

Index Terms-Constructive interference, Multi-antenna receiver, Imperfect channel estimation, Robust precoder

\section{INTRODUCTION}

Multiple-input and multiple-output (MIMO) has been widely considered as a key technology in 5G communications, due to its significant gains over single-antenna configuration [1]. When downlink users are equipped with multiple receivers $(\mathrm{RX}) \mathrm{s}$, a multiuser MIMO system is formed and there are two flavours of MIMO design with respect to how data is transmitted across the given channel, namely spatial multiplexing or diversity. In spatial multiplexing, each spatial channel carries independent information and thus each user receives multi-layer data streams [1]. On the other hand, spatial diversity scheme aims at improving the transmission reliability, and base station (BS) sends one-layer data (same data) for each user across different propagation (spatial) paths [2]. Due to its high reliability against deep channel fading, spatial diversity based multiuser MIMO has been acknowledged as the most practical MIMO technique and has attracted much attention in both academia and industry.

With the channel state information (CSI) available at the BS and users, joint combiner and precoder design becomes feasible in multiuser MIMO systems. To be specific, each user combines the data from its multiple RXs, and hence the precoder at the BS is developed to generate orthogonal transmission among the multiple users by suppressing multiuser interference. Over the past few years, the capacity achieving dirty-paper coding scheme was proposed in [3] by pre-subtracting the interference

\footnotetext{
Zhongxiang Wei, Christos Masouros and Tongyang $\mathrm{Xu}$ are with the department of Electronic and Electrical Engineering at the University College London, London, UK. Email: \{zhongxiang.wei, c.masouros, tongyang.xu.11\}@ucl.ac.uk
}

prior to transmission, which is difficult to implement due to the impractical assumption of infinite alphabet and its high computational cost. To strike performance and complexity, non-linear Tomlinson-Harashima precoding [4] and vector perturbation [5] were proposed, which however are still too complicated in practice due to the inclusion of the sophisticated sphere-search algorithms. To this end, low-complexity linear precoders based on zero-forcing (ZF) [6] and minimum mean squared error (MMSE) precoders [7] have been implemented as practical solutions. On the other hand, downlink precoding schemes based on optimization have also been a popular research topic. One form of the optimization known as signal-to-interferenceplus-noise ratio (SINR) balancing is to maximize the minimum SINR subject to power constraint [8]. An alternative downlink precoding strategy targets at minimizing the total transmit power at the BS subject to multiple users' minimum SINR requirements [9]. In summary, the principle of existing designs is to mitigate multiuser interference by exploiting channel correlation.

The above designs are only optimal from a statistical viewpoint, where the transmitted signal is treated as infinite Gaussian signal. Since the correlation among the transmitted signal and channel is not effectively exploited, multiuser interference has to be mitigated as much as possible. However, interference is not always destructive [10]. It is because in practice, modulation size is limited and input signal is not Gaussian signal. One can rotate, rather than mitigating, the correlation of the transmitted data, so that the interfering signal is able to be aligned to the signal of interest for enhancing system performance, referred to as constructive interference (CI). CI yields received signals that have increased distances from the decision thresholds of the constellation compared to the nominal constellation points. In other words, to achieve a target receive SINR at the users, less transmission power is consumed by utilizing interference over the conventional schemes that cancel interference. The concept of CI was firstly introduced by [10] in code division multiple access. Then a rotated ZF-based scheme was proposed in [11] to partially utilize interference, and [12] further proposed that all the interference can be constructive by precoder design operating at symbol level. Recently, CI was applied into generic multiple-input and singleoutput (MISO) systems [13] [14] [15], large-scale MIMO [16], hybrid beamforming [17], multi-cell coordination [18], and 
physical layer security [19].

To date, CI technique is only investigated for MISO configuration where each user is equipped with single antenna, and its application in multi-receiver user scenario is left untouched. The fundamental challenges lie in 1) Since the symbols to be transmitted are naturally known by BS, CI precoder is enabled to exploit the correlation among the channels and symbols for multiuser interference utilization. Nevertheless, downink users are only aware of their channel but have no knowledge of the intended symbols. As a result, the design information at the BS and users turns to be asymmetric. How to jointly design combiner at the users to compatize CI precoder at the transmitter is still unknown. 2) Considering a practical imperfect CSI scenario at both BS and users, it requires robust joint CI combiner and precoder design against CSI error. More importantly, the performance degradation of the CI technique caused by the imperfect CSI needs to be fully evaluated. 3) Considering practical modulation and coding schemes (MCS)s standardized by IEEE protocols, does the implementation of cyclic redundancy check (CRC) and low-density parity-check (LDPC) coding dissolve the advantage of CI precoder? This again requires fundamental analysis for the CI related design. To address the open issues, we are motivated to investigate joint CI combiner and precoder design in multiuser MIMO systems. Our contribution can be summarized as:

1) To the best of our knowledge, it is the first work explicitly investigating joint $\mathrm{CI}$ combiner and precoder design for multi-RX users scenario. To be specific, the combiner of each user is designed based on the largest singular value of its MIMO channel, which is mostly likely to be used to carry the signal-of-interest for each user due to the high channel gain. Subsequently, precoder is developed to exploit the correlation among the channels and the transmitted symbols, which keeps the users' composite leakage (multiuser inference) constructive at each user.

2) We then investigate a practical scenario where CSI acquisition at the $\mathrm{BS}$ and users is not perfect. Leveraging the statistical distribution of CSI, CSI error bound is first derived. Then, a deterministic manner robust precoder is developed, which always guarantees the CI condition even in the worst CSI case.

3) Finally, the multiple users' receive performance is analyzed under the effect of CRC and LDPC channel coding, proving the superiority of the proposed joint CI combiner and precoder design with the standardized protocol.

Notations: Matrices and vectors are represented by boldface capital and lower case letters, respectively. $|\cdot|$ denotes the absolute value. $\|\cdot\|_{F}$ denotes the Frobenius norm. $(\cdot)^{H}$ and $(\cdot)^{T}$ denote the Hermitian transpose and transpose operation. Operator $\Re$ and $\Im$ return the real and imaginary parts of a complex number, respectively. $\boldsymbol{I}_{n}$ denotes a $n$-by- $n$ identity matrix. $\mathbb{C}^{N \times M}$ denotes the set of $N \times M$ matrix.

\section{SYSTEM MODEL}

We investigate joint combiner and CI precoder design in multiuser MIMO systems at downlink, where the MIMO diversity scheme is adopted. Without loss of generality, the BS equipped with $N_{t}$ transmit antennas is simultaneously communicating with $K$ users in the same time- and frequency-resource, where $K \leq N_{t}$. Each user is equipped with $N_{r}$ RXs. Denote $\boldsymbol{H}_{k} \in \mathbb{C}^{N_{r} \times N_{t}}$ as flat-fading Rayleigh channel from the BS to the $k$-th user, and $\boldsymbol{W} \in \mathbb{C}^{N_{t} \times K}$ as precoder employed at the BS. Denoting $s \in \mathbb{C}^{K \times 1}$ as the transmitted symbol, the received signal at the $k$-th user is expressed as

$$
\boldsymbol{r}_{k}=\boldsymbol{H}_{k} \boldsymbol{W} s+\boldsymbol{n}_{k}, \forall k \in K,
$$

where $\boldsymbol{n}_{k} \in \mathbb{C}^{N_{r} \times 1}$ is additive Gaussian noise following distribution $\boldsymbol{n}_{k} \sim \mathbb{C N}\left(\mathbf{0}, \sigma_{k}^{2} \boldsymbol{I}_{N_{r}}\right), \forall k \in K$. With a combiner $\boldsymbol{c}_{k} \in \mathbb{C}^{1 \times N_{r}}$ at the $k$-th user, the post-combined signal is equalized into

$$
y_{k}=\boldsymbol{c}_{k} \boldsymbol{r}_{k}=\boldsymbol{c}_{k} \boldsymbol{H}_{k} \boldsymbol{W} \boldsymbol{s}+\boldsymbol{c}_{k} \boldsymbol{n}_{k}
$$

which is finally used for demodulation and decoding purpose.

\section{Fundamentals of CI AND PROBlem Formulation}

Conventional MIMO techniques mitigate multiuser interference as a harmful element, thus containing each user's received signal within a region around the nominal point in the modulated signal constellation, as illustrated in Fig. 1(a). Differently, $\mathrm{CI}$ is the interference that pushes the received signal away from the detection threshold of the signal demodulation, and hence the intended signal of each user falls into constructive regions (green area) of Fig. 1(b) [18]. To show this intuitively, assume a desired symbol $s_{k}=\frac{1}{\sqrt{2}}+\frac{1}{\sqrt{2}} j$ of the $k$-th user, from a normalized QPSK constellation for illustration purpose. As observed in Fig. 1(c), to make the $k$-th user's desired signal $y_{k}$ (which could be the post-combined signal) located in the constructive region, we have to guarantee the rotated signal $y_{k} s_{k}^{*}$ satisfy the following inequality such that

$$
\left.\mid \Im\left\{y_{k} s_{k}^{*}\right)\right\} \mid \leq\left(\Re\left\{y_{k} s_{k}^{*}\right)-d_{k}\right\} \tan \theta, \forall k \in K .
$$

where the operators $\Re$ and $\Im$ denote the real and imaginary parts of a complex value, respectively. $\theta=\pi / M$ with $M$ denoting the constellation size. Evidently, $d$ physically represents the Euclidean distance in the signal constellation between the $k$ th user's received signal and the decision thresholds, and thus a larger value of $d$ represents a higher receive SINR performance.

On the understanding of the fundamentals of CI, we are able to present problem formulation, where combiner and precoder are jointly designed to maximize the minimum value of $d_{k}$, such as 


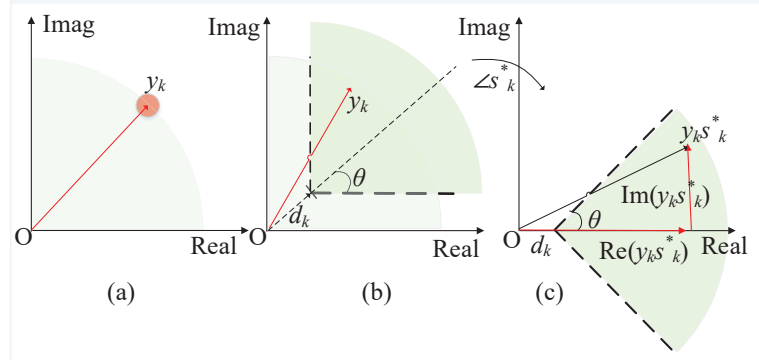

Fig. 1. QPSK for illustration. (a) Conventional precoder: the intended signal is expected to be in a proximity region around the constellation point and any multiuser interference adds perturbation to the signal. (b) CI technique: interference pushes the symbol away from the original decision thresholds of the constellation, and the desired signal $y_{k}$ falls into constructive region (green area). (c) Rotated by $\angle s_{k}^{*}, \Re\left\{y_{k} s_{k}^{*}\right\}$ and $\Im\left\{y_{k} s_{k}^{*}\right\}$ are mapped onto real and imaginary axis. Hence, the trigonometric interpretation of CI can be exploited, as shown by Eq. (3).

$$
\begin{aligned}
& P 1: \underset{\boldsymbol{W}, \boldsymbol{c}_{k}}{\operatorname{argmax}} \min d_{k}, \\
& \left.(C 1): \mid \Im\left\{y_{k} s_{k}^{*}\right)\right\} \mid \leq\left(\Re\left\{y_{k} s_{k}^{*}\right)-d_{k}\right\} \tan \theta, \forall k \in K, \\
& (C 2):\|\boldsymbol{W}\|_{F}^{2} \leq P_{\max },
\end{aligned}
$$

where $(\mathrm{C} 1)$ generates the constructive multiuser interference at each user, while (C2) makes the transmission power lower than a budget $P_{\max }$.

\section{JOINT CI BASEd COMBINER AND CI PRECODER DESIGN}

CI precoder exploits both the channel and input signal's correlation to utilize multiuser interference, while the input signal is not known by the downlink users. Due to the asymmetry of design information at the BS and users, the design of precoder and combiner in P1 has to be decoupled, i.e., designing combiner first and then presenting precoder with the equivalent channels. A simple combiner design at the users can be an equal-gain combiner such that $\boldsymbol{c}_{k}=\mathbf{1} \in \mathbb{C}^{1 \times N_{r}}$, where 1 denotes a vector with all-1-element. Evidently, by the equal-gain combiner, CSI is not necessarily to be known at the users with reduced overhead. Differently, combiner can be designed based on the singular-value-decomposition (SVD) of $\boldsymbol{H}_{k}$, which achieves superior performance over the equal-gain combiner. Write the SVD operation of $\boldsymbol{H}_{k}$ as

$$
\boldsymbol{H}_{k}=\boldsymbol{U}_{k} \boldsymbol{\Lambda}_{k} \boldsymbol{V}_{k}^{H}, \forall k \in K,
$$

where $\boldsymbol{U}_{k}$ and $\boldsymbol{V}_{k}$ are unitary-matrices. Write $\boldsymbol{U}_{k}$ as

$$
\boldsymbol{U}_{k}=\left[\boldsymbol{u}_{k 1}, \ldots, \boldsymbol{u}_{k N_{r}}\right],
$$

where $\boldsymbol{u}_{k n} \in \mathbb{C}^{N_{r} \times 1}$ denote the $n$-th column of $\boldsymbol{U}_{k}$. At the $k$-th user, it uses the Hermitian of first column of $\boldsymbol{U}_{k}$ as its combiner, i.e., $\boldsymbol{c}_{k}=\boldsymbol{u}_{k 1}^{H}$. Physically, it corresponds the largest singular vector of the channel $\boldsymbol{H}_{k}$, and is mostly likely to be used to carry the signal-of-interest for the $k$-th user. Hence, the spatial diversity of the largest singular value based combiner is enhanced over the equal-gain combiner. Now, the post-combined signal is rewritten as

$$
y_{k}=\boldsymbol{h}_{k} \boldsymbol{W} \boldsymbol{s}+\boldsymbol{c}_{k} \boldsymbol{n}_{k},
$$

where $\boldsymbol{h}_{k}=\boldsymbol{u}_{k 1} \boldsymbol{H}_{k} \in \mathbb{C}^{1 \times N_{t}}$ denotes the equivalent MISO channel from the BS to the $k$-th user. Now, we can turn to handle precoder design. As aforementioned, conventional precoders need to suppress multiuser interference as much as possible. Hence, the $k$-th user's signal is transmitted close to the null space of other users' channels, which in fact may be far from the high gain channel $\boldsymbol{h}_{k}$. Thereby, it reduces the degrees-of-freedom (DoF) of precoder design. Differently, when delivering the $k$-th user's signal, CI precoder is not necessary to transmit signal along the orthogonal space of others' channel, but still concentrates high transmission power on the space that is close to $\boldsymbol{h}_{k}$ and simultaneously keeps all the other users' inference constructive at the $k$-th user, $\forall k \in K$. Accordingly, the superiority of the CI based joint combiner and precoder design is essentially built on delivering the signalof-interest along the channel that the users has high channel gain while treating their leakage as a constructive element. For ease of expression, we write $\boldsymbol{W}=\left[\boldsymbol{w}_{1}, \boldsymbol{w}_{2}, \ldots, \boldsymbol{w}_{K}\right]$, where $\boldsymbol{w}_{k} \in \mathbb{C}^{N_{t} \times 1}$ denotes the precoder vector for the $k$-th user's desired symbol $s_{k}$. Hence, the received signal at the $k$-th user is re-written as

$$
y_{k}=\boldsymbol{h}_{k} \sum_{i=1}^{K} \boldsymbol{w}_{i} s_{i}+\boldsymbol{c}_{k} \boldsymbol{n}_{k} .
$$

Since the BS knows the symbol $s_{k}=e^{j \phi_{k}}$ (symbol amplitude is normalized without loss of generality), $s_{k}$ can be written as a rotated version of another symbol such as $s_{k}=s_{i} e^{j\left(\phi_{k}-\phi_{i}\right)}$. To this end, the received signal in (8) can be re-formulated as

$$
y_{k}=\boldsymbol{h}_{k} \sum_{i=1}^{K} \boldsymbol{w}_{i}\left(s_{k} e^{j\left(\phi_{i}-\phi_{k}\right)}\right)+\boldsymbol{c}_{k} \boldsymbol{n}_{k} .
$$

Without loss of generality, we select the symbol $s_{1}=d e^{j \phi_{1}}$ as the reference. (9) is equivalent to

$$
\begin{aligned}
y_{k} & =\boldsymbol{h}_{k} e^{j\left(\phi_{1}-\phi_{k}\right)} \sum_{i=1}^{K} \boldsymbol{w}_{i} e^{j\left(\phi_{i}-\phi_{1}\right)} s_{k}+\boldsymbol{c}_{k} \boldsymbol{n}_{k} \\
& =\boldsymbol{g}_{k} \boldsymbol{t} s_{k}+\boldsymbol{c}_{k} \boldsymbol{n}_{k},
\end{aligned}
$$

where $\boldsymbol{g}_{k}=\boldsymbol{h}_{k} e^{j\left(\phi_{1}-\phi_{k}\right)}$ and $\boldsymbol{t}=\sum_{i=1}^{K} \boldsymbol{w}_{i} e^{j\left(\phi_{i}-\phi_{1}\right)}$. The reformulation in (10) indicates that the original broadcast channel reduces to a virtual multicast channel with common 
symbols to all users. Aided by (10), CI condition in (3) can be equivalently written as

$$
\left|\Im\left\{\boldsymbol{g}_{k} \boldsymbol{t}\right\}\right| \leq\left(\Re\left\{\boldsymbol{g}_{k} \boldsymbol{t}\right)-d_{k}\right\} \tan \theta, \forall k \in K,
$$

which locates the resulting interfered signals in the constructive regions. Now, optimization problem P1 is readily given in the form of

$$
\begin{aligned}
& P 2: \underset{\boldsymbol{t}, \boldsymbol{c}_{k}}{\operatorname{argmax}} \min d_{k}, \\
& (C 1):\left|\Im\left\{\boldsymbol{g}_{k} \boldsymbol{t}\right\}\right| \leq\left(\Re\left\{\boldsymbol{g}_{k} \boldsymbol{t}\right)-d_{k}\right\} \tan \theta, \forall k \in K, \\
& (C 2):\|\boldsymbol{t}\|_{F}^{2} \leq P_{\max },
\end{aligned}
$$

which is a standard convex function and can be readily solved by CVX. With the obtained optimal $t^{*}$, it is not difficult to give the optimal precoders as

$$
\boldsymbol{w}_{1}^{*}=\frac{\boldsymbol{t}^{*}}{K}, \text { and } \boldsymbol{w}_{k}^{*}=\frac{\boldsymbol{t}^{*} e^{j\left(\phi_{1}-\phi_{k}\right)}}{K} .
$$

which finally yields the optimal solution of the CI-based joint combiner and precoder (CI-CP) design.

\section{Robust CI Combiner AND PRECoder Design with IMPERFECT CSI}

In practice, ideal CSI at the BS and users may not be obtained. Therefore, the robust manner joint combiner and precoder need to be accordingly designated, and the impact of non-ideal CSI on the performance of CI design should be fully evaluated. In this section, we further propose robust CI-based joint combiner and precoder (Robust-CI-CP), taking into consideration of imperfect CSI acquisition. Imperfect CSI model and problem formulation are first given in subsection $\mathrm{V}-\mathrm{A}$, and then its solution is introduced in V-B.

\section{A. Imperfect CSI Model and Problem Formulation}

Due to the asymmetry of design information at the BS and users, robust combiner at the users may not be achieved without the information of the transmitted symbols from the BS. Hence with imperfect CSI, the combiner $\boldsymbol{c}_{k}$ also can be obtained based on the estimated version of $\boldsymbol{H}_{k}$. In addition, it is fact that by un-biased channel estimators, the expectation of the estimated channel approaches the real channel, and thus utilizing the estimated channel for designating combiner achieves a nearoptimality. With the effect of the combiner, a series of similar derivations in (8)-(11) can be employed, and the MIMO channel $\boldsymbol{H}_{k}$ is equivalently reduced to a MISO channel $\boldsymbol{g}_{k}$ indicated by (10). With CSI error, it is written as

$$
\boldsymbol{g}_{k}=\hat{\boldsymbol{g}}_{k}+\boldsymbol{e}_{k},
$$

where $\hat{\boldsymbol{g}}_{k} \in \mathbb{C}^{1 \times N_{t}}$ and $\boldsymbol{e}_{k} \in \mathbb{C}^{1 \times N_{t}}$ represent the estimated channel and estimation error [20], respectively. In particular, the error vector $\boldsymbol{e}_{k}$ follows i.i.d Gaussian distribution, i.e., $\boldsymbol{e}_{k} \sim \mathbb{C N}\left(\mathbf{0}, \sigma_{e}^{2} \boldsymbol{I}_{N_{t}}\right)$. Again, we attempt to optimize receive performance by jointly designing combiner and precoder under the effect of imperfect CSI, where the problem formulation is given as

$$
\begin{aligned}
& \text { P3 : } \underset{\boldsymbol{t}}{\operatorname{argmax}} \min d_{k} \text {, } \\
& \text { (C3): } \left.\max _{\left\|\boldsymbol{e}_{k}\right\|_{F}^{2} \leq \zeta^{2}} \mid \Im\left\{\left(\hat{\boldsymbol{g}}_{k}+\boldsymbol{e}_{k}\right) \boldsymbol{t}\right)\right\} \mid \leq \\
& \left.\Re\left\{\left(\hat{\boldsymbol{g}}_{k}+\boldsymbol{e}_{k}\right) \boldsymbol{t}\right)-d_{k}\right\} \tan \theta, \forall k \in K, \\
& \text { (C4) : }\|\boldsymbol{t}\|_{F}^{2} \leq P_{\text {max }},
\end{aligned}
$$

where (C3) guarantees the CI exploitation under the worst case of CSI acquisition, i.e., the CSI error reaching its uncertainty bound $\zeta^{2}$. (C4) makes power lower than the budget $P_{\max }$.

\section{B. Optimization Solution}

In P3, it can be seen that (C3) contains infinite CSI uncertainties. Hence, we first introduce Lemma 1 for obtaining the CSI error bound $\zeta^{2}$, which specifies an ellipsoidal uncertainty region for the estimated CSI.

Lemma 1: Given that the element of CSI error follows Gaussian distribution such that $\left[\boldsymbol{e}_{k}\right]_{n} \sim \mathbb{C N}\left(0, \sigma_{e}^{2}\right)$, the value of $\left\|e_{k}\right\|_{F}^{2}$ is approximately upper bounded by $\zeta^{2}=\frac{\sigma_{e}^{2} \Phi_{2 N_{t}}^{-1}(\eta)}{2}$. In particular, $\eta$ physically represents the outage probability of $\left\|\boldsymbol{e}_{k}\right\|_{F}^{2}$ violating its upper bound $\zeta^{2}$, which can be set close to 1, i.e., $\eta=0.9$. $\Phi_{2 N_{t}}^{-1}(\cdot)$ is the inverse function of the cdf of a chi-square distributed variable with $2 N_{t}$ degrees of freedom. $\square$

Proof: Please see Appendix A.

Aided by Lemma 1, we can re-formulate the optimization problem $\mathrm{P} 3$ as

$$
\begin{aligned}
& P 4: \underset{t}{\operatorname{argmax}} \min d_{k}, \\
& \left.(C 3): \max _{\left\|e_{k}\right\|_{F}^{2} \leq \zeta^{2}} \mid \Im\left\{\left(\hat{\boldsymbol{g}}_{k}+\boldsymbol{e}_{k}\right) \boldsymbol{t}\right)\right\} \mid \leq \\
& \left.\Re\left\{\left(\hat{\boldsymbol{g}}_{k}+\boldsymbol{e}_{k}\right) \boldsymbol{t}\right)-d_{k}\right\} \tan \theta, \forall k \in K, \\
& (C 4):\|\boldsymbol{t}\|_{F}^{2} \leq P_{\max },(C 5): \zeta^{2}=\frac{\sigma_{e}^{2} \Phi_{2 N_{t}}^{-1}(\eta)}{2}, \forall k \in K \text {. }
\end{aligned}
$$

We now define real-valued error vector and channel estimation vector as

$\overline{\boldsymbol{e}}_{k}=\left[\boldsymbol{e}_{k}^{\Re}, \boldsymbol{e}_{k}^{\Im}\right], \overline{\boldsymbol{g}}_{k}=\left[\hat{\boldsymbol{g}}_{k}^{\Re}, \hat{\boldsymbol{g}}_{k}^{\Im}\right], \boldsymbol{t}_{1}=\left[\boldsymbol{t}^{\Im}, \boldsymbol{t}^{\Re}\right]$, and $\boldsymbol{t}_{2}=\left[\boldsymbol{t}^{\Re},-\boldsymbol{t}^{\Im}\right]$.

With these notations, we can re-express the real and imaginary parts in (C3) as follows

$$
\Im\left\{\boldsymbol{g}_{k} \boldsymbol{t}\right\}=\overline{\boldsymbol{g}}_{k} \boldsymbol{t}_{1}+\overline{\boldsymbol{e}}_{k} \boldsymbol{t}_{1} \text {, and } \Re\left\{\boldsymbol{g}_{k} \boldsymbol{t}\right\}=\overline{\boldsymbol{g}}_{k} \boldsymbol{t}_{2}+\overline{\boldsymbol{e}}_{k} \boldsymbol{t}_{2},
$$

As a consequence, constraint (C3) can be decoupled into 

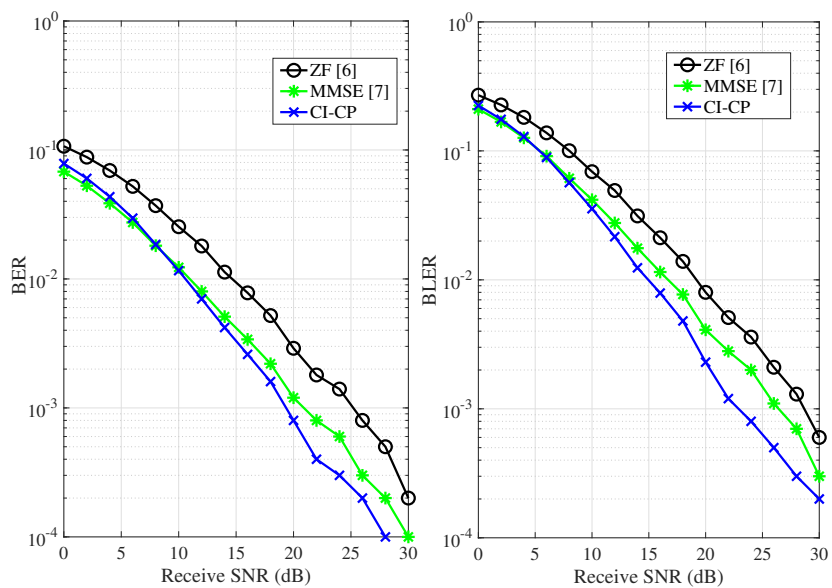

Fig. 2. The impact of receive SNR (in $\mathrm{dB}$ ) on the BER and BLER performance by different designs.

$$
\begin{aligned}
& \max _{\left\|\boldsymbol{e}_{k}\right\|_{F}^{2} \leq \zeta^{2}} \overline{\boldsymbol{g}}_{k} \boldsymbol{t}_{1}+\overline{\boldsymbol{e}}_{k} \boldsymbol{t}_{1}-\left(\overline{\boldsymbol{g}}_{k} \boldsymbol{t}_{2}+\overline{\boldsymbol{e}}_{k} \boldsymbol{t}_{2}\right) \tan \theta+d_{k} \tan \theta \leq 0, \\
& \text { and } \\
& \max _{\left\|\boldsymbol{e}_{k}\right\|_{F}^{2} \leq \zeta^{2}}-\overline{\boldsymbol{g}}_{k} \boldsymbol{t}_{1}-\overline{\boldsymbol{e}}_{k} \boldsymbol{t}_{1}-\left(\overline{\boldsymbol{g}}_{k} \boldsymbol{t}_{2}+\overline{\boldsymbol{e}}_{k} \boldsymbol{t}_{2}\right) \tan \theta+d_{k} \tan \theta \leq 0,
\end{aligned}
$$

whose robust formulations are given by

$$
\begin{gathered}
\overline{\boldsymbol{g}}_{k} \boldsymbol{t}_{1}-\overline{\boldsymbol{g}}_{k} \boldsymbol{t}_{2} \tan \theta+\zeta\left\|\boldsymbol{t}_{1}-\boldsymbol{t}_{2} \tan \theta\right\|_{F}+d_{k} \tan \theta \leq 0, \text { and } \\
-\overline{\boldsymbol{g}}_{k} \boldsymbol{t}_{1}-\overline{\boldsymbol{g}}_{k} \boldsymbol{t}_{2} \tan \theta+\zeta\left\|\boldsymbol{t}_{1}-\boldsymbol{t}_{2} \tan \theta\right\|_{F}+d_{k} \tan \theta \leq 0,
\end{gathered}
$$

Now P3 is a standard convex optimization problem and can be solved by CVX directly, which finally yields the solution of the Robust-CI-CP design.

\section{Vi. Simulation Results}

We present the Monte-Carlo simulation results in this section. It is assumed that a typical lamp-size BS is equipped with 4 antennas. There are 4 users, each equipped with 4 receive antennas. CRC and LDPC chain standardized by the MCS index 15 of 3GPP TS 38.214 is adopted, with 616/1024 code rate and QPSK modulation. The symbol vector $s$ is randomly generated. Rayleigh fading channel is adopted. Without loss of generality, power budget $P_{\max }$ is normalized to 1 . The linear ZF [6] and MMSE [7] precoders are selected as benchmarks, where their combiner is calculated by the largest singular vector of the MIMO channel.

In Fig. 2, we compare the bit error rate (BER) and block error rate (BLER) performance of the different designs, with ideal CSI acquisition. It can be seen that the proposed CI-CP design always outperforms the ZF with all SNRs, and is superior over the MMSE at above $6 \mathrm{~dB}$ SNR regimes. It is because by the proposed design, the rich multiuser interference is utilized a
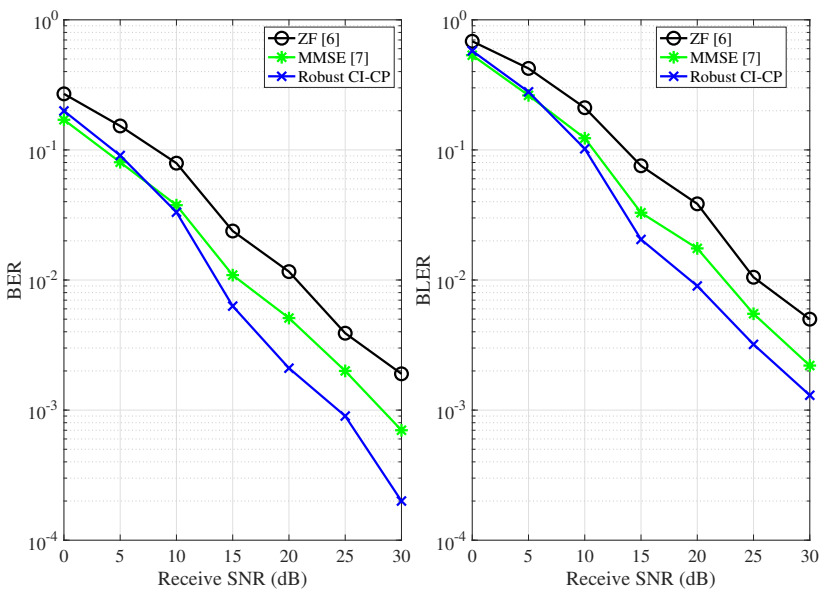

Fig. 3. The impact of receive SNR (in $\mathrm{dB}$ ) on the BER and BLER performance by different designs, with imperfect CSI acquisition.

beneficial element rather than being strictly suppressed. As a result, the required SNR achieving 0.01 BLER decreases $2 \mathrm{~dB}$ compared to MMSE precoder, and $4 \mathrm{~dB}$ compared to $\mathrm{ZF}$ precoder. In Fig. 3, we demonstrate the BER and BLER performance of the different designs, with a moderate CSI quality $\sigma_{e}^{2}=10^{-3}$. It is observed that the proposed robust CI design always outperforms ZF precoder at any SNR regimes, and achieves lower BLER over MMSE precoder with above $5 \mathrm{~dB}$ SNR, showing a higher level of robustness against CSI error.

\section{CONCLUSION}

In this work, we have investigated CI-based joint combiner and precoder design for multiuser MIMO systems. Due to the asymmetric design information at the BS and users, combiner at each user is first performed with the largest singular value of its MIMO channel, and subsequently CI precoder is designed to utilize multiuser interference as a beneficial element. In addition, a practical imperfect CSI acquisition scenario is considered. A deterministic-type robust combiner and precoder design is developed against CSI error, which guarantees the CI condition in the worst case CSI acquisition. Finally, simulation results have revealed the superior performance of the proposed design in terms of BER and BLER.

\section{APPENDIX A \\ PROOF OF LEMMA 1}

The term $\left\|e_{k}\right\|_{F}^{2} \leq \zeta^{2}$ can be equivalently written as $\left\|\frac{\Re\left\{\left[\boldsymbol{e}_{k}\right]_{1}\right\}}{\sigma_{e} / \sqrt{2}}+\frac{\Im\left\{\left[\boldsymbol{e}_{k}\right]_{1}\right\}}{\sigma_{e} / \sqrt{2}}+\ldots+\frac{\Re\left\{\left[\boldsymbol{e}_{k}\right]_{N_{t}}\right\}}{\sigma_{e} / \sqrt{2}}+\frac{\Im\left\{\left[\boldsymbol{e}_{k}\right]_{N_{t}}\right\}}{\sigma_{e} / \sqrt{2}}\right\|_{F}^{2} \leq \zeta^{2}$. The left hand follows chi-square distribution with $2 N_{t}$ degrees of freedom. Hence, $\left\|e_{k}\right\|_{F}^{2} \leq \zeta^{2}$ can be approximately interpreted as the pdf of a chi-square variable with $2 N_{t}$ degrees of freedom such that 


$$
\begin{aligned}
\operatorname{Pr}\left\{\left(\frac{\Re\left\{\left[\boldsymbol{e}_{k}\right]_{1}\right\}}{\sigma_{e} / \sqrt{2}}\right)^{2}+\left(\frac{\Im\left\{\left[\boldsymbol{e}_{k}\right]_{1}\right\}}{\sigma_{e} / \sqrt{2}}\right)^{2}+\ldots+\right. \\
\left.\quad\left(\frac{\Re\left\{\left[\boldsymbol{e}_{k}\right]_{N_{t}}\right\}}{\sigma_{e} / \sqrt{2}}\right)^{2}+\left(\frac{\Im\left\{\left[\boldsymbol{e}_{k}\right]_{N_{t}}\right\}}{\sigma_{e} / \sqrt{2}}\right)^{2} \leq\left(\frac{\zeta}{\sigma_{e} / \sqrt{2}}\right)^{2}\right\}=\eta,
\end{aligned}
$$

where $\eta$ physically represents the probability of the inequality being satisfied [18]. Evidently, (21) can be regarded as a cdf such that $\Phi_{2 N_{t}}\left(\frac{\zeta^{2}}{\sigma_{e}^{2} / 2}\right)=\eta$, where $\Phi_{2 N_{t}}(\cdot)$ is the cdf of a chisquare variable with $2 N_{t}$ degrees of freedom. Defining $\Phi_{2 N_{t}}^{-1}(\cdot)$ as its inverse function, finally we get $\zeta^{2}=\frac{\sigma_{e}^{2} \Phi_{2 N_{t}}^{-1}(\eta)}{2}$.

\section{REFERENCES}

[1] F. Sohrabi and W. Yu, "Hybrid digital and analog beamforming design for large-scale antenna arrays," IEEE J. Sel. Topics Sig. Proc., vol. 10, no. 3, pp. 501-513, Apr. 2016

[2] R. W. Heath and A. J. Paulraj, "Switching between diversity and multiplexing in MIMO systems," IEEE Trans. on Commun., vol. 53, no. 6, pp. 962-968, Jun. 2005.

[3] M. Costa, "Writing on dirty paper," IEEE Trans. Inf. Theory, vol. 29, no. 3, pp. 439-441, May 1983.

[4] L. Sun and M. Lei, "Quantized CSI-based Tomlinson-Harashima precoding in multiuser MIMO systems," IEEE Trans. Wireless Commun., vol. 12, no. 3, pp. 1118-1126, Mar. 2013.

[5] B. M. Hochwald, C. B. Peel, and A. L. Swindlehurst, "A vectorperturbation technique for near-capacity multiantenna multiuser communication-Part II: Perturbation," IEEE Trans. Commun., vol. 53, no. 3, pp. 537-544, Mar. 2005.

[6] A. Wiesel, Y. C. Eldar, and S. Shamai, "Zero-forcing precoding and generalized inverses," IEEE Trans. Signal Process., vol. 56, no. 9, pp. 4409-4418, Sep. 2008.

[7] K. Zhu, C. Lamare, and M. Haardt, "Generalized design of lowcomplexity block diagonalization type precoding algorithms for multiuser MIMO systems," IEEE Trans. Commun., vol. 61, no. 10, pp. 4232-4242, Oct. 2013.
[8] M. F. Hanif, L.-N. Tran, A. Tolli, and M. Juntti, "Computationally efficient robust beamforming for SINR balancing in multicell downlink with applications to large antenna array systems" IEEE Trans. Commun., vol. 62, no. 6, pp. 1908-1920, Jun. 2014.

[9] N. D. Sidiropoulos, T. N. Davidson, and Z.-Q. Luo, "Transmit beamforming for physical-layer multicasting", IEEE Trans. Signal Process., vol. 54, no. 6, pp. 2239-2251, Jun. 2006.

[10] C. Masouros and E. Alsusa, "A Novel Transmitter-Based SelectivePrecoding Technique for DS/CDMA systems", IEEE Signal Processing Lett., vol. 14, no. 9, pp. 637-640, Sept. 2007.

[11] C. Masouros, E. Alsusa, "Dynamic linear precoding for the exploitation of known interference in MIMO broadcast systems", IEEE Trans. Wireless Comms., vol. 8, no. 3, pp. 1396-1404, Mar. 2009.

[12] C. Masouros, "Correlation rotation linear precoding for MIMO broadcast communications", IEEE Trans. Sig. Proc., vol. 59, no. 1, pp. 252-262, Jan. 2011.

[13] C. Masouros, M. Sellathurai, and T. Ratnarajah, "Vector perturbation based on symbol scaling for limited feedback MISO downlinks," IEEE Trans. Sig. Proc., vol. 62, no. 2, pp. 562-571, Feb. 2014.

[14] D. Spano, M. Alodeh, S. Chatzinotas, and B. Ottersten, "Symbol level precoding for the non-linear multiuser MISO downlink channel," IEEE Trans. Signal Process., vol. 66, no. 5, pp. 1331-1345, Mar. 2018.

[15] M. Alodeh, S. Chatzinotas, and B. Ottersten, "Constructive multiuser interference in symbol level precoding for the MISO downlink channel," IEEE Trans. Signal Process., vol. 63, no. 9, pp. 2239-2252, May 2015.

[16] S. Domouchtsidis, C. G. Tsinos, S. Chatzinotas, and B. Ottersten, "Symbol-level precoding for low complexity transmitter architectures in large-scale antenna array systems," IEEE Trans. Wireless Commun., vol. 18, no. 2, pp. 852-863, Feb. 2019.

[17] G. Hegde, C. Masouros, M. Pesavento, "Coordinated Hybrid Precoding for Interference Exploitation in Heterogeneous Networks", IEEE Commun. Lett., vol. 23, no. 11, Nov. 2019.

[18] Z. Wei, C. Masouros, K. Wong, and X. Kang, "Multi-cell interference exploitation: a new dimension in cell coordination", IEEE Trans. Wireless Commun., vol. 19, no. 1, pp. 1303-1312, Oct. 2019.

[19] Z. Wei, F. Liu and C. Masouros, "Secure directional modulation with few-bit phase shifters: optimal and iterative-closed-form designs", IEEE Trans. Commun., DOI: 10.1109/TCOMM.2020.3032459

[20] S. Boyd, Stanford University [2008, Winter Quarter] Linear dynamical systems, [Online] Available: https://stanford.edu/class/ee363/lectures/estim.pdf 